Revista de Investigación en Psicología. Vol. 2, № 1. pp. 73 - 90

\title{
LA AUTOESTIMA Y LOS VALORES EN LAS MICROEMPRESAS DEL CONO ESTE DE LIMA
}

\author{
Alejandro Loli Pineda \\ Ernestina López Vega \\ María Atalaya Pisco
}

Se investigó sobre la jerarquía de valores que impera en las microempresas del Cono Este de Lima, la relación entre la autoestima y los valores organizacionales, y las diferencias entre la autoestima y algunos rasgos demográficos, así como éstos con los valores organizacionales. El estudio se llevó a cabo en una muestra de 105 gerentes de igual número de microempresas. La información fue recabada mediante el Inventario de Autoestima (ALPEL) Forma-AD y la Lista de Valores Organizacionales (ALPEL) Forma VA. El análisis de los resultados nos indica que sí existe una jerarquía de valores, si bien de muy baja importancia, pero de los más elegidos. Igualmente, existe correlación positiva y moderada de la autoestima alta con lealtad y amor al trabajo, y una correlación negativa de la autoestima baja con lealtad y el trabajo en equipo. Por otro lado, la correlación entre la autoestima y los valores organizaciones con rasgos demográficos vana sustancialmente.

Palabras clave: Jerarquía, autoestima, valores organizacionales, microempresa.

It was investigated on the values hierarchy that prevails in the small business of the east zone of Lima, the relationship between self-esteem and the organizational values. and the differences between the self-esteem and some demographic features, as well as these with the organizational values. The study was carried out in a sample of 105 managers of equal number o small business. The information was collected through the Inventory of self-esteem (ALPEL) VA-Form. and the Values Organizational List (ALPEL) VA-Form. The analysis o/the results indicate us that indeed exists a hierarchy of values. even though of very little importance, but of the most elected. Equally, it exists positive correlation and moderated other high self-esteem with loyalty and love to the work, and a negative correlation of the low self-esteem with loyalty and the work in team. On the other side, the correlation between self-esteem and so me organizational values. with demographic features there are more differences.

Key words: Hierarchy, self-esteem, organizational values, microenterprise. 
Los estudios desarrollados en tomo a la autoestima y los valores coinciden en creer que estos dos aspectos de la personalidad se inician a muy temprana edad. La autoestima según Freud tiene sus orígenes en la etapa oral (primeros 18 meses), donde surge la crisis de transición al pasar de la "confianza habitual" a la "desconfianza", es aquel momento donde el niño recibe todo lo que le da y no tiene capacidad para dar, entre tanto Erick Erikson (1950) ratificando la teoría Freudiana, considera que la autoestima se origina en la etapa sensorio-oral (de 1 a 2 años), allí donde se definen la confianza y la desconfianza, dependiendo de la estabilidad y continuidad de atención que los padres ofrecen al infante; es decir, durante la formación de la estructura psíquica sobre la base del "ello", cuando el niño ("yo") descubre al otro ("tu") y que es diferente a él. Vandenbergh (1963), señala el desarrollo de la confianza en base a comprensión y amor (reforzando el amor a si mismo). De allí que Rogers (1969) encuentre que la raíz de gran cantidad de problemas radica en que se desprecian y se consideran seres sin valor, indignos de ser amados, teoría sobre la cual se basa todo el constructo psicoterapéutico rogeriano.

Los valores, igualmente, tienen su origen en el proceso de formación de la estructura psíquica, donde sobre la base del "ello", el "yo" se relaciona con el mundo exterior y nace la necesidad de establecer las primeras reglas de convivencia humana (entre 18 y 24 meses), llamado también la conciencia moral o "super yo", haciendo que el niño se transforme en un ser moral (es capaz de controlar sus esfínteres y pedir la satisfacción de algunas necesidades). Bela Szekely (1963) decía, en tomo al tema, que el "ello es la parte animal del hombre", el "yo" es el que relaciona con el otro y el "super yo" es el representante de la sociedad. Así nace uno de los primeros valores en la vida del hombre (la moral) prolongándose a lo largo de su existencia y en donde subyacen todos los demás, dispuestos a los requerimientos del "yo" en su interacción con el mundo.

En términos de Piaget (1970), los primeros sentimientos morales surgen del respeto unilateral del niño hacia los padres o el adulto; respeto que provoca la "formación de una moral de obediencia ... " a lo que llama también "sentimientos morales intuitivos", y que van acompañados de regulaciones de intereses y valores relacionados al pensamiento intuitivo. 
Como se ve, por experiencias propias, transmitidas o intervenciones impuestas, se dan situaciones en la vida de las personas aceptadas o rechazadas en armonía con los mandatos de la conciencia, reafirmándose el compromiso con la sociedad. Así las personas involucradas en la sociedad aprenden a encarar las condiciones sociales, económicas y políticas que se les presentan.

Estudios realizados en 43 de las empresas más brillantes de Estados Unidos de Norteamérica (Thomas J. Peters y Robert H. Waterman, 1982), nos informan de ocho factores como clave del éxito y grandeza de estas empresas, de los cuales seis estaban vinculados a la naturaleza humana y todos éstos tenían que ver con los valores. Por otro lado, las investigaciones basadas en la Ley de Yerkes Dobson $\left(1975\right.$ - 1990) ${ }^{1}$ señalan que la autoestima reforzada es capaz de elevar la productividad y calidad de un trabajador e ir mucho más allá para mejorar su actitud en el servicio, llevando además la autoestima y el cambio personal hasta sentirse orgulloso, satisfecho con un empleo.

Este cambio de percepción laboral solo es posible (Yerkes, 1978) con el desarrollo de la autoestima hasta el nivel que Maslow (1952) señala como nivel de autorrealización personal (1. Cruz, 1996). Juan de la Poza y Juan Prior (1988), estudiaron a 80 servidores de la salud en una Clínica de la ciudad de Granada - España y encontraron que la autoestima en el trabajo "juega un papel modulador en la relación entre clima organizacional y satisfacción con el trabajo", similar a lo encontrado por Mossholder (1981) y Adler (1980).

Nuestro interés por conocer nuestra realidad DOS llevó al planteamiento de una serie de interrogaciones, que finalmente quedaron así: ¿Cuál es la jerarquía de valores que imperan en microempresas de Lima?, ¿qué relación existe entre la autoestima y los valores organizacionales? y ¿qué diferencias existen entre la autoestima y los valores organizacionales en relación con 1 a procedencia, edad, sexo, estado civil, grado de instrucción

1 La Ley Yerkes (1978) afirma que “... hay un nivel óptimo de excitación para desempeñar cualquier tarea de la mejor manera posible; mientras más compleja sea ésta, menor será el nivel de excitación tolerable antes de que el desempeño se deteriore". 
y actividades económico-comerciales de la empresa?

Si es cierto que la autoestima, especialmente los valores varían de persona a persona, de empresa a empresa y de sociedad a sociedad en armonía con la cultura que ellas poseen, aun así, éstas han contribuido al éxito de las empresas en muchas de ellas, incluso ya se habla de la "admiración por valores" (Blanchard y O'Connor, 1997). Por consiguiente, es posible que en las organizaciones empresariales de nuestro país:

a) Los valores organizacionales positivos son los que imperan en la jerarquía de valores de las empresas.

b) Existe relación entre la autoestima de los propietarios, gerentes o administradores y los valores organizacionales de las empresas que dirigen.

c) Existen diferencias entre la autoestima de los propietarios, gerentes o administradores de las empresas en relación con su procedencia, edad, sexo, estado civil, grado de instrucción y actividades económico comerciales de la empresa.

d) Existen diferencias entre los valores organizacionales jerarquizados por los propietarios, gerentes o administradores de las empresas en relación con su procedencia, edad, sexo, estado civil, grado de instrucción y las actividades económico-comerciales de la empresa.

\section{METODOLOGÍA}

La metodología empleada es la descriptiva comparativa entre la autoestima y los valores organizacionales. Para determinar esta relación se empleó la correlación de Pearson; mientras que para ver las diferencias entre la autoestima y algunas variables (mayores a dos grupos) se recurrió al Análisis de Varianza. Cuando se trató de ver las diferencias entre otras variables (dos grupos) se usó el puntaje $t$ de Student.

La muestra estuvo conformada por propietarios, gerentes o administradores de 105 microempresas que constituyen el 23\% de un total de 450, pertenecientes a seis asociaciones de empresarios unidos en una organización de nivel superior, ubicados en el Cono Este (distrito de Ate - Vitarte) de Lima Metropolitana. 
La técnica empleada para la recolección de la información fue la entrevista individual estructurada a cada uno de los integrantes de la muestra en el lugar de trabajo, mediante la prueba de Autoestima y valores Organizacionales.

En la investigación se empleo el Inventario de Autoestima (ALPEL), Forma - AD y la Lista de Valores Organizacionales (ALPEL), Forma VA, ambos instrumentos fueron construidos con fines exclusivos de la presente investigación y aplicados después de una validación de su consistencia interna mediante el sistema de jueces, y que en el momento actual se trabaja en la ampliación de la muestra para darle el tratamiento estadístico que permita la confiabilidad del instrumento.

\section{RESULTADOS}

\section{Jerarquizacion de valores organizacionales}

La Tabla 1 nos nuestra los 26 valores organizacionales puestos en prueba y la forma cómo se distribuyen a opinión de los miembros de la muestra; siendo los 10 más importantes aquellos de más baja valoración. Es decir, cuanto más alto es el número de sujetos que la eligen, más bajo es el valor que le otorgan, excepto Liderazgo Transparente, que es elegido por siete, pero todos lo valoran mejor o lo ubican en una mejor posición; en cambio, Puntualidad es uno de los valores más elegidos entre los diez más importantes, sin embargo, pocos le otorgan la importancia correspondiente; tal vez porque a pesar de ser importante dicho valor, no es tomado muy en cuenta en la práctica organizacional. De todos modos, estos valores son positivos y están presentes en las microempresas.

\section{Autoestima y valores organizacionales}

La correlación entre la Autoestima Alta y los Valores Organizacionales (Tabla 2) es moderada y positiva con Lealtad; la cual indica que cuanto más alta es la autoestima, más importante es la lealtad $\left(0.47^{*}\right)$; mientras que la correlación con Amor al Trabajo, cuanto más alta es la autoestima, 
menor es la valoración de amor al trabajo (- 0.27*).

La correlación entre la Autoestima Baja y los valores Organizacionales (Tabla 2) es negativa con lealtad; es decir, que cuanto más baja es la autoestima hay menos lealtad (- $0.40 *)$; igualmente, cuanto más baja es la autoestima, menos se valora el trabajo en equipo (- $\left.0.38^{*}\right)$. Con lo que se confirma en parte la relación entre la autoestima de los propietarios, gerentes o administradores y algunos valores organizacionales que señala la segunda hipótesis, porque no existe correlación significativa entre la autoestima y los demás valores organizacionales.

\section{Tabla 1 \\ Jerarquización de los valores organizacionales}

\begin{tabular}{|l|l|c|c|c|}
\hline ORDEN & VALORES ORGANIZACIONALES & M & DS & CASOS \\
\hline & Puntualidad & 3.08 & 2.69 & 88 \\
02 & Limpieza & 4.13 & 2.82 & 64 \\
03 & Calidad Del Producto & 4.25 & 2.63 & 57 \\
04 & Lealtad & 4.33 & 2.41 & 30 \\
05 & Responsabilidad & 4.36 & 2.74 & 91 \\
06 & Honradez & 4.50 & 2.34 & 86 \\
07 & Liderazgo Transparente & 4.86 & 2.48 & 7 \\
08 & Amor Al Trabajo & 5.31 & 2.79 & 55 \\
09 & Trabajo En Equipo & 5.35 & 2.42 & 40 \\
10 & Creatividad & 5.47 & 2.38 & 36 \\
11 & Respeto & 5.73 & 2.70 & 66 \\
12 & Ahorro & 6.09 & 2.05 & 34 \\
13 & Disciplina & 6.31 & 2.60 & 49 \\
14 & Cortesía & 6.36 & 1.99 & 28 \\
\hline
\end{tabular}

(*) Significativo $\mathrm{p}<.05$

\section{La autoestima y algunos rasgos demográficos}

Las Tablas 3 al 9 muestran la comparación de los niveles de autoestima con diversos rasgos demográficos (región de procedencia, lugar de procedencia, edad, género, estado civil) mediante el estadístico $t$ de Student, y con grado de instrucción y actividades de la empresa, mediante el Análisis de 
Varianza. Los resultados nos indican que no existen diferencias significativas en esa relación, lo que significa que la autoestima mantiene inalterable frente a dicha variable.

Tabla 2

Comparación entre los valores organizacionales y niveles de autoestima

\begin{tabular}{|l|c|c|}
\hline VALORES ORGANIZACIONALES & \multicolumn{2}{|c|}{ AUTOESTIMA } \\
\hline & ALTA (r) & BAJA (r) \\
& & \\
Puntualidad & 0.01 & -0.03 \\
Limpieza & 0.23 & -0.22 \\
Lealtad & $0.47^{*}$ & $-0.40^{*}$ \\
Calidad del Producto & -0.03 & -0.13 \\
Trabajo en Equipo & 0.06 & $-0.38^{*}$ \\
Honradez & 0.02 & -0.01 \\
Ahorro & 0.17 & -0.23 \\
Comunicación Abierta & -0.14 & 0.09 \\
Liderazgo Transparente & -0.35 & 0.35 \\
Creatividad & 0.03 & -0.02 \\
Justicia & 0.21 & -0.30 \\
Cortesía & -0.29 & -0.29 \\
Disciplina & -0.08 & 0.08 \\
Responsabilidad & -0.08 & 0.14 \\
Orden & -0.03 & 0.03 \\
Respeto a la Persona & -0.14 & 0.16 \\
Ética & -0.16 & 0.16 \\
Deseo de Superación & -0.04 & -0.02 \\
Amor al Trabajo & -0.27 & 0.11 \\
Gestión Participativa & 0.65 & -0.65 \\
Información a Todo Nivel & -0.18 & -0.09 \\
Iniciativa & -0.46 & 0.46 \\
Servicio al Cliente & -0.08 & 0.15 \\
Confianza & -0.01 & 0.19 \\
Moralidad & -0.19 & -0.10 \\
Armonía & 0.10 & \\
\hline
\end{tabular}

(*) Significativo $\mathrm{p}<.05$ 
Tabla 3

Comparación de los niveles de autoestima según género

\begin{tabular}{|c|c|c|c|}
\hline \multirow{2}{*}{ AUTOESTIMA } & \multicolumn{2}{|c|}{ GENERO } & \multirow{2}{*}{ "t" } \\
\cline { 2 - 3 } & VARON & MUJER & \\
\cline { 2 - 3 } & $\mathrm{N}=79$ & $\mathrm{~N}=26$ & -0.40 \\
\hline Alta & 35.22 & 35.69 & 0.24 \\
\hline Baja & 10.59 & 10.30 & \\
\hline
\end{tabular}

(*) Significativo $\mathrm{p}<$. O5

Tabla 4

Comparación de los niveles de autoestima según estado civil

\begin{tabular}{|c|c|c|c|}
\hline \multirow{2}{*}{ AUTOESTIMA } & \multicolumn{2}{|c|}{ ESTADO CIVIL } & \multirow{2}{*}{ " $\mathrm{t}$ " } \\
\cline { 2 - 3 } & SOLTERO & CASADO & \\
\cline { 2 - 3 } & $\mathrm{N}=34$ & $\mathrm{~N}=63$ & 0.41 \\
\hline Alta & 35.58 & 35.12 & $-0.21(*)$ \\
\hline Baja & 10.41 & 10.65 & \\
\hline
\end{tabular}

Significativo $\mathrm{p}<.05$

Tabla 5

Comparación de los niveles de autoestima según grado de instrucción

\begin{tabular}{|c|c|c|c|c|}
\hline \multirow{2}{*}{ AUTOESTIMA } & \multicolumn{3}{|c|}{ GRADO DE INSTRUCCIÓN } & \multirow{2}{*}{$\mathrm{F}$} \\
\cline { 2 - 4 } & PRIMARIA & SECUND & SUPERIOR & \\
\cline { 2 - 4 } & $\mathrm{N}=12$ & $\mathrm{~N}=62$ & $\mathrm{~N}=31$ & 2.60 \\
\hline Alta & 33.50 & 34.90 & 36.93 & 2.21 \\
\hline Baja & 12.50 & 10.87 & 9.06 & \\
\hline
\end{tabular}

(*) Significativo $\mathrm{p}<.05$ 
Tabla 6

Comparación de los niveles de autoestima según grupo de edad

\begin{tabular}{|c|c|c|c|c|}
\hline \multirow{3}{*}{ AUTOESTIMA } & \multicolumn{3}{|c|}{ EDAD } & \multirow{2}{*}{$\mathrm{F}$} \\
\cline { 2 - 4 } & $\begin{array}{c}18-30 \text { años } \\
\mathrm{N}-32\end{array}$ & $\begin{array}{c}31-40 \text { años } \\
\mathrm{N}=32\end{array}$ & $\begin{array}{c}41 \text { a más } \\
\mathrm{N}=41\end{array}$ & \\
\hline Alta & 35.12 & 35.18 & 35.63 & 0.10 \\
\hline Baja & 10.87 & 11.65 & 9.36 & 1.82 \\
\hline
\end{tabular}

(*) Significativo $\mathrm{p}<.05$

Tabla 7

Comparación de los niveles de autoestima según la región de procedencia

\begin{tabular}{|c|c|c|c|}
\hline \multirow{2}{*}{ AUTOESTIMA } & \multicolumn{2}{|c|}{ REGION DE PROCEDENCIA } & \multirow{2}{*}{ "t" } \\
\cline { 2 - 4 } & $\begin{array}{c}\text { COSTA } \\
\mathrm{N}=32\end{array}$ & $\begin{array}{c}\text { SIERRA } \\
\mathrm{N}=70\end{array}$ & \\
\hline Alta & 34.50 & 35.85 & -1.25 \\
\hline Baja & 10.21 & 10.10 & 0.14 \\
\hline
\end{tabular}

(*) Significativo $\mathrm{p}<.05$

Tabla 8

Comparación de los niveles de autoestima según el lugar de procedencia

\begin{tabular}{|c|c|c|c|}
\hline \multirow{2}{*}{ AUTOESTIMA } & \multicolumn{2}{|c|}{ LUGAR DE PROCEDENCIA } & \multirow{2}{*}{ "t" } \\
\cline { 2 - 4 } & $\begin{array}{c}\text { LIMA y CALLAO } \\
\mathrm{N}=19\end{array}$ & $\begin{array}{c}\text { PROVINC } \\
\mathrm{N}=5\end{array}$ & \\
\hline Alta & 34.15 & 33.40 & 0.18 \\
\hline Baja & 9.68 & 12.60 & -1.22 \\
\hline
\end{tabular}

(*) Significativo $\mathrm{p}<.05$ 


\section{Tabla 9 \\ Comparación de los niveles de autoestima según actividades económicas}

\begin{tabular}{|l|c|c|c|}
\hline \multirow{2}{*}{\begin{tabular}{c}
\multirow{2}{*}{$\begin{array}{c}\text { ACTIVIDADES } \\
\text { ECONOMICAS }\end{array}$} \\
\cline { 2 - 3 }
\end{tabular}} & \multicolumn{2}{c|}{ AUTOESTIMA } & \multirow{2}{*}{$\mathrm{N}$} \\
\cline { 2 - 3 } Servicio & 35.12 & 10.87 & 16 \\
\hline Comercio & 34.69 & 9.73 & 23 \\
\hline Carpintería de Madera & 35.00 & 11.00 & 18 \\
\hline Carpintería Metálica & 36.14 & 11.42 & 14 \\
\hline Metal Mecánica & 35.86 & 10.13 & 15 \\
\hline Fundición & 36.50 & 9.50 & 08 \\
\hline Juegos Educativos & 39.00 & 7.00 & 01 \\
\hline Calzados & 34.00 & 12.00 & 04 \\
\hline Confecciones & 33.50 & 12.50 & 02 \\
\hline Artesanía & 35.75 & 10.25 & 04 \\
\hline F & 0.25 & 0.26 & \\
\hline
\end{tabular}

(*) Significativo $\mathrm{p}<.05$

\section{Los valores organizacionales y algunos rasgos demográficos}

Al comparar los Valores Organizacionales según Género (Tabla 10) se observa que existe diferencia significativa en "Creatividad", la cual es mejor valorada por los varones que por las mujeres $(-2.21 *)$ como valores importantes de sus empresas; mientras que en el "Servicio al Cliente" de parte de sus organizaciones, las mujeres le asignan mayor valor que los varones $\left(2.15^{*}\right)$.

De la comparación entre los Valores Organizacionales de acuerdo con el Grado de Instrucción (Tabla 11) se observa que existen diferencias significativas tanto en "limpieza" (3.16*) como en "disciplina" (3.24*). Encontrándose que en ambos casos la valoración más relevante corresponde a los ejecutivos que tienen estudios de primaria, seguidos por los que tienen estudios de secundaria y finalmente por los que tienen estudios superiores. Es decir, a mayor nivel de educación formal menos importancia a los Valores Organizacionales de limpieza y disciplina. 
Tabla 10

Comparación de los valores organizacionales según género

\begin{tabular}{|c|c|c|c|}
\hline \multirow[b]{2}{*}{ VALORES ORGANIZACIONALES } & \multicolumn{3}{|c|}{ GENERO } \\
\hline & $\begin{array}{c}\text { VARON } \\
N=79\end{array}$ & $\begin{array}{c}\text { MUJER } \\
\mathrm{N}=26\end{array}$ & "t" \\
\hline Puntualidad & 3.04 & 3.18 & -0.20 \\
\hline Limpieza & 4.54 & 3.05 & 1.94 \\
\hline Lealtad & 4.10 & 4.80 & -0.74 \\
\hline Calidad del Producto & 4.02 & 5.62 & -1.62 \\
\hline Trabajo en Equipo & 5.25 & 6.00 & -0.64 \\
\hline Honradez & 4.71 & 4.00 & 1.31 \\
\hline Ahorro & 6.19 & 5.92 & 0.36 \\
\hline Comunicación Abierta & 6.52 & 6.44 & 0.11 \\
\hline Liderazgo Transparente & 4.80 & 5.00 & -0.09 \\
\hline Creatividad & 5.10 & 7.33 & $-2.21^{*}$ \\
\hline Justicia & 6.90 & 8.57 & -1.46 \\
\hline Cortes fa & .05 & 7.00 & -1.19 \\
\hline Disciplina & 6.61 & 5.46 & 1.38 \\
\hline Responsabilidad & 4.37 & 4.31 & 0.09 \\
\hline Orden & 6.96 & 6.30 & 0.65 \\
\hline Respeto a la Persona & 5.45 & 6.35 & -0.24 \\
\hline Etica & 7.16 & 8.00 & -0.88 \\
\hline Deseo de Superación & 6.48 & 6.92 & -0.50 \\
\hline Amor al Trabajo & .31 & 5.27 & 0.05 \\
\hline Gestión Participativa & 8.25 & 10.00 & -3.13 \\
\hline Información a Todo Nivel & 7.81 & 9.00 & -0.86 \\
\hline Iniciativa & 6.37 & 7.00 & -0.31 \\
\hline Servicio al Cliente & 6.93 & 4.90 & $2.15 *$ \\
\hline Confianza & 7.83 & 7.66 & 0.18 \\
\hline Moralidad & 8.88 & 7.33 & 1.01 \\
\hline Armonfa & 6.44 & 8.50 & -0.03 \\
\hline
\end{tabular}

(*) Significativo $\mathrm{p}<.05$

Al comparar los Valores Organizacionales según Lugar de Procedencia (Tabla 12) se observa que existe diferencia significativa sólo en el caso del valor organizacional "Cortesía" (- 2.44*), en el cual, las empresas dirigidas por sujetos procedentes de Lima y Callao estarían tomando más en cuenta la cortesía como característica de actuación de sus empresas que los de 
provincia. En los demás, no existen diferencias, son similares. De la comparación entre los Valores Organizacionales

\section{Tabla 11}

Comparación de los valores organizacionales según grado de instrucción

\begin{tabular}{|l|c|c|c|c|}
\hline \multirow{2}{*}{ VALORES ORGANIZACIONALES } & \multicolumn{3}{|c|}{ GRADO DE INSTRUCION } & \\
& PRIM. & SECUND. & SUP & F \\
& N=12 & N=62 & N=31 & \\
\hline Puntualidad & 4.10 & 2.70 & 3.40 & 1.42 \\
Limpieza & 2.50 & 3.71 & 5.17 & $3.16^{*}$ \\
Lealtad & 5.75 & 3.94 & 4.50 & 0.93 \\
Calidad del Producto & 5.12 & 4.13 & 4.00 & 0.52 \\
Trabajo en Equipo & 6.25 & 5.52 & $4: 86$ & 0.61 \\
Honradez & 4.44 & 4.42 & 4.68 & 0.10 \\
Ahorro & 5.66 & 6.04 & 6.33 & 0.12 \\
Comunicación Abierta & 5.66 & 6.41 & 7.16 & 068 \\
Liderazgo Transparente & 4.50 & 6.33 & 300 & 1.18 \\
Creatividad & 4.00 & 5.63 & 5.54 & 0.61 \\
Justicia & 8.75 & 7.90 & 4.66 & 363 \\
Cortesía & 8.66 & 6.25 & 5.92 & 2.64 \\
Disciplina & 4.00 & 6.43 & 7.07 & $3.24 *$ \\
Responsabilidad & 4.09 & 4.33 & 4.53 & 0.10 \\
Orden & 5.33 & 6.83 & 7.11 & 0.48 \\
Respeto A La Persona & 6.44 & 5.64 & 5.55 & 0.36 \\
Etica & 9.00 & 7.00 & 7.60 & 1.12 \\
Deseo De Superación & 5.62 & 6.84 & 6.41 & 063 \\
Amor al Trabajo & 4.14 & 5.70 & 5.05 & 1.00 \\
Gestión Participativa & ----- & 9.00 & 8.00 & 1.80 \\
Información a Todo Nivel & 10.0 & 8.25 & 7.40 & 0.67 \\
Iniciativa & 7.33 & 600 & 6.50 & 0.25 \\
Servicio al Cliente & 8.25 & 6.84 & 5.80 & 1.67 \\
Confianza & 7.80 & 8.11 & 6.75 & 1.03 \\
Moralidad & 10.0 & 8.60 & 8.16 & 0.23 \\
Armonía & 8.00 & 900 & 6.50 & 0.96 \\
\hline
\end{tabular}

(*) Significativo $\mathrm{p}<.05$

según la Actividad Económica de las empresas (Tabla 13) se observa que existen diferencias significativas en el valor "justicia" (3.41*), .la misma que es mejor valorado por los sujetos que realizan actividades en empresas de Fundición y Carpintería en Madera que aquellos que realizan otras actividades; también hay diferencias en el valor "información a todo nivel" $(4.57 *)$, siendo las personas de empresas que brindan Servicio las que 
mejor califican este valor, en comparación de aquellas que se desempeñan en el Comercio, Carpintería en Madera, Metal Mecánica, Carpintería Metálica, y Fundición. En otros términos, las actividades económicas a las que se dedican los ejecutivos de las empresas determinan, de alguna manera, las diferencias de valores en sus empresas.

Tabla 12

Comparación de los valores organizacionales según lugar de procedencia

\begin{tabular}{|c|c|c|c|}
\hline \multirow[b]{2}{*}{$\begin{array}{l}\text { VALORES } \\
\text { ORGANIZACIONALES }\end{array}$} & \multicolumn{2}{|c|}{ LUGAR DE PROCEDENCIA } & \multirow[b]{2}{*}{ "t" } \\
\hline & $\begin{array}{l}\text { LIMA y CALLAO } \\
\qquad \mathrm{N}=19\end{array}$ & $\begin{array}{l}\text { PROVINCIA } \\
\quad \mathrm{N}=5\end{array}$ & \\
\hline Puntualidad & 2.70 & 2.33 & 0.23 \\
\hline Limpieza & 3.83 & 3.00 & 0.57 \\
\hline Lealtad & 3.85 & 2.00 & 0.64 \\
\hline Calidad del Producto & 4.66 & 3.00 & 0.90 \\
\hline Trabajo en Equipo & 4.85 & 5.33 & -0.24 \\
\hline Honradez & 3.87 & 4.60 & -0.88 \\
\hline Ahorro & 6.80 & 5.00 & 0.66 \\
\hline Comunicación Abierta & 6.83 & 6.00 & 0.58 \\
\hline Liderazgo Transparente & ---- & ------ & ----- \\
\hline Creatividad & 6.00 & 7.00 & -0.49 \\
\hline Justicia & 5.00 & ----- & ------ \\
\hline Cortesía & 5.20 & 8.00 & $-2.44 *$ \\
\hline Disciplina & 7.42 & 6.00 & -0.23 \\
\hline Responsabilidad & 4.83 & 4.80 & 0.02 \\
\hline Orden & 7.28 & 3.50 & 1.98 \\
\hline Respeto a la Persona & 4.90 & 6.60 & -1.02 \\
\hline Etica & 7.50 & 10.00 & -1.41 \\
\hline Deseo de Superación & 7.09 & 8.33 & -0.71 \\
\hline Amor al Trabajo & 6.30 & 4.00 & 1.05 \\
\hline Gestión Participativa & 8.00 & ----- & ----- \\
\hline Información a Todo Nivel & 8.50 & ----- & ----- \\
\hline Iniciativa & 6.75 & 9.50 & -1.38 \\
\hline Servicio al Cliente & 7.16 & 8.00 & -0.41 \\
\hline Confianza & 7.80 & 4.00 & 1.34 \\
\hline Moralidad & 8.50 & 10.00 & -0.58 \\
\hline Armonía & 10.00 & ----- & ----- \\
\hline
\end{tabular}

(*) Significativo $\mathrm{p}<.05$ 
Por consiguiente, encontramos que entre algunos valores organizacionales y variables tales como lugar de procedencia, género, grado de instrucción y actividades de la empresa se presentan diferencias significativas, excepto cuando se relaciona con estado civil, edad y región de procedencia; por tanto, se confirma en parte la tercera hipótesis.

\section{DISCUSIÓN}

Los resultados obtenidos, en términos generales, nos confirman la presencia de la autoestima y los valores en las organizaciones. Tanto la autoestima como los valores surgen en el hombre y lo acompañan en el curso de su vida, transmitiendo e influyendo en la conformación de rasgos psíquicos en grupos y organizaciones

Todos tenemos una jerarquía de valores que constituyen nuestro sistema de valores. "El sistema se identifica en razón de la importancia que se conceda a los valores ... " (Robbins, 1994), "representan la convicción básica de que una forma de conducta especifica o de condición última de la vida son preferibles, en términos personales o sociales a otra forma de conducta contrarias u opuestas" (Rokeach, 1973) y son de vital importancia en la conducta organizacional. Nuestros resultados confirman la presencia de esta jerarquía al que se refiere Robbins, simplemente, que no todos los que lo eligen como los adecuados para sus empresas, le conceden la misma importancia, pero las prefieren (por ejemplo, la puntualidad, limpieza, lealtad, calidad del producto, trabajo en equipo, honradez, ahorro, comunicación abierta, liderazgo transparente, creatividad) a otra forma de conducta contraria; tal vez porque a pesar de ser importantes dichos valores, alguno de ellos es menos tomado en cuenta en la práctica organizacional.

Sin embargo, al comparar los valores organizacionales con los niveles de autoestima de los ejecutivos de las microempresas, sí existe correlación con algunos valores (lealtad, amor al trabajo, trabajo en equipo) confirmándose en parte nuestros supuestos, y es probable que esta relación mejore con investigaciones en muestras más amplias, porque entre la autoestima y los valores existe una correspondencia: 


\section{Tabla 13 \\ Comparación de los valores organizacionales según actividad económica}

\begin{tabular}{|c|c|c|c|c|c|c|c|c|c|c|c|}
\hline \multirow[t]{2}{*}{$\begin{array}{c}\text { VALORES } \\
\text { ORGANIZACIONALES }\end{array}$} & \multicolumn{11}{|c|}{$\begin{array}{l}\text { ACTIVIDADES } \\
\text { ECONÓMICAS }\end{array}$} \\
\hline & Serv. & Comerc. & $\begin{array}{l}\text { Carp } \\
\text { Mad }\end{array}$ & $\begin{array}{l}\text { Carp } \\
\text { Met }\end{array}$ & $\begin{array}{l}\text { Met. } \\
\text { Mec }\end{array}$ & Fund & $\begin{array}{l}\text { Juegs } \\
\text { Educ }\end{array}$ & Calz & Conf & Art. & $\mathrm{F}$ \\
\hline & $\mathrm{N}=16$ & $\mathrm{~N}=23$ & $\mathrm{~N}=18$ & $\mathrm{~N}=14$ & $\mathrm{~N}=15$ & $\mathrm{~N}=8$ & $\mathrm{~N}=\mathrm{I}$ & $\mathrm{N}=4$ & $\mathrm{~N}=2$ & $\mathrm{~N}=4$ & \\
\hline Puntualidad & 3.50 & 2.65 & 3.62 & 1.83 & 4.00 & 4.57 & 1.00 & 1.50 & 1.00 & 2.00 & 1.20 \\
\hline limpieza & 3.64 & 3.63 & 5.12 & 5.14 & 4.25 & 6.75 & ---- & 2.66 & 8.00 & 2.00 & 1.54 \\
\hline Lealtad & 4.33 & 3.75 & 5.80 & 3.00 & 5.80 & 3.50 & ---- & ----- & 300 & 2.00 & 0.97 \\
\hline Calidad del Product. & 4.25 & 3.83 & 4.45 & 3.45 & 4.60 & 3.00 & 10.0 & 4.00 & 1.00 & 9.00 & 1.92 \\
\hline Trabajo en equipo & 6.50 & 5.00 & 4.33 & 4.85 & 6.37 & 6.00 & 9.00 & 3.00 & ---- & 4.50 & 1.06 \\
\hline Honradez & 4.46 & 4.52 & 5.61 & 4.09 & 4.75 & 4.00 & 2.00 & 4.00 & 3.00 & 3.75 & 0.66 \\
\hline Ahorro & 5.50 & 5.81 & 7.00 & 6.50 & 5.33 & 6.00 & ---- & ---- & 9.00 & 5.00 & 0.62 \\
\hline Comunico Abierta & 6.60 & 8.20 & 6.66 & 6.33 & 5.00 & 4.00 & ----- & 6.00 & ----- & 6.00 & 1.26 \\
\hline Liderazgo Transp. & 4.00 & 5.50 & 9.00 & 5.00 & ----- & 1.00 & ----- & ----- & ----- & ---- & 687 \\
\hline Creatividad & 5.75 & 6.00 & 5.57 & 7.50 & 3.66 & 4.33 & ---- & 5.50 & 4.00 & 7.00 & 1.04 \\
\hline Justicia & 8.00 & 8.60 & 4.50 & 7.66 & 10.00 & 4.00 & ----- & ----- & ---- & 9.00 & $3.41^{\prime}$ \\
\hline Cortesia & 5.40 & 6.77 & 5.00 & 7.66 & 8.33 & 4.50 & ----- & ----- & 5.00 & 8.00 & 1.85 \\
\hline Disciplina & 6.44 & 6.09 & 6.50 & 5.66 & 6.33 & 5.66 & ---- & 7.00 & 10.0 & 5.00 & 0.36 \\
\hline Responsabilidad & 5.61 & 4.77 & 3.86 & 5.00 & 2.64 & 4.20 & 6.00 & 4.25 & 4.50 & 4.00 & 1.15 \\
\hline Orden & 7.50 & 7.50 & 6.12 & 4.33 & 6.00 & 6.50 & 8.00 & 9.00 & 7.00 & 7.00 & 0.57 \\
\hline Respeto a la Persona & 4.72 & 6.42 & 3.70 & 6.00 & 7.16 & 4.66 & & 6.66 & 6.50 & 6.00 & 1.71 \\
\hline Etica & 8.25 & 8.66 & 6.33 & 5.00 & 8.00 & 8.33 & 5.00 & ---- & 5.00 & & 2.15 \\
\hline Deseo de Superación & 6.75 & 6.80 & 6.69 & 7.10 & 6.10 & 5.33 & ---- & 9.00 & 9.00 & 4.66 & 0.64 \\
\hline Amor al Trabajo & 5.00 & 6.36 & 4.69 & 440 & 4.87 & 6.00 & 4.00 & 7.00 & -.--- & 6.00 & 0.51 \\
\hline Gestión Participativa & ---- & ----- & 8.00 & 8.00 & 9.00 & 8.00 & -.--- & ---- & .--- & 10.0 & ----- \\
\hline Lnform. a todo nivel & 4.00 & 9.00 & 10.00 & 8.25 & 9.00 & 8.00 & ----- & ---- & 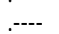 & ----. & $4.57^{*}$ \\
\hline Iniciativa & 4.00 & 8.33 & 7.25 & 5.00 & 5.33 & 6.66 & ---. & 7.00 & ---- & ---- & 0.64 \\
\hline Servicio al Cliente & 5.12 & 5.84 & 6.00 & 8.62 & 6.83 & 7.37 & 3.00 & 9.00 & 5.00 & 8.00 & 1.51 \\
\hline Confianza & 8.00 & 7.28 & 7.75 & 7.71 & 7.75 & 9.00 & ---- & 7.50 & ---- & 9.00 & 0.14 \\
\hline Moralidad & 8.00 & 7.50 & 8.66 & 10.00 & 10.00 & 10.00 & 7.00 & ---- & ----- & ---- & 0.20 \\
\hline Armonía & 9.00 & 4.00 & 8.25 & 9.00 & 9.00 & ---.- & .--.- & 10.0 & -.--- & 10.0 & 0.64 \\
\hline
\end{tabular}

(*)significalivo $\mathrm{p}<.05$

"cuando la autoestima es alta los valores son positivos, e inversamente, cuando la autoestima es baja los valores son negativos". La disonancia de esta correspondencia genera actitudes y conductas anómalas (Loli y López, 1997) tanto en lo personal como en lo organizacional. Por ello, si bien aún no es posible una afirmación empírica en el sentido que la llave de la calidad y el éxito del hombre se encuentra esencialmente en la autoestima (Loli y López, 1997) y no sólo en los valores como afirma Rodríguez (1993); es innegable, sin embargo, que el "punto de partida en la inductiva de los valores (positivos) sea el incremento de la" (Mora, 1996). Probablemente éstas sean las razones por las que la autoestima en 
el trabajo "juega un papel modulador en la relación entre el clima organizacional y la satisfacción con el trabajo" (De la Poza y Prior, 1988; Mossholder, 1981; Adler, 1980); naturalmente, éstas no son sino consecuencia de la interacción entre los niveles de autoestima y los valores organizacionales ofrecidos por los representantes claves de la organización.

Sin embargo, cuando se comparan los niveles de autoestima con algunos rasgos demográficos (edad, genero, procedencia, estado civil, grado de instrucción y actividad económica de la empresa) de los ejecutivos de las empresas no hay diferencias significativas; es decir, la autoestima se mantiene inalterable frente a la presencia de dichas variables. Pero cuando se comparan los valores organizacionales con dichas variables encontramos algunas diferencias significativas, especialmente en relación al lugar de procedencia, genero, grado de instrucción y actividades de la empresa, probablemente porque existen diferentes expectativas (a partir de las influencias y experiencias culturales del entorno organizacional) o son más sensibles. Esto explicaría porqué las empresas recurren a situaciones vedadas para solucionar problemas organizacionales (que aparentemente no comprometen los valores personales de sus ejecutivos). Mientras la autoestima infunde energía, pasión, visión, etc., a la persona o al conjunto, los valores personales controlan y regulan su actuación (Mora, 1996); luego, lo positivo o negativo del conjunto -si bien con influencia personal de sus miembros- su percepción y actuación dependerán de las experiencias y oportunidades permitidas por su entorno social económico y cultural.

\section{CONCLUSIONES}

Los resultados del análisis estadístico y bibliográfico nos permiten arribar a algunas conclusiones:

a) Los valores organizacionales más bajos corresponden a los ítemes más elegidos por propietarios, gerentes o administradores (ejecutivos) de las microempresas estudiadas, tal vez porque a pesar de ser importantes dichos valores para ellos, son menos considerados en la práctica organizacional. 
b) La correlación entre Autoestima Alta y los Valores Organizacionales es moderada y positiva con algunos valores: Cuanta más alta es la autoestima más importante es la lealtad, pero menos importante es el amor al trabajo. La correlación entre la Autoestima Baja y los Valores Organizacionales es negativa con algunos valores: Cuanto más baja es la autoestima menos importante es la lealtad; y también se le otorga menor valor al trabajo en equipo.

e) La autoestima en relación con algunas variables demográficas analizadas (procedencia, edad, sexo, estado civil, y grado de instrucción) indican que no existen diferencias significativas, de manera que la autoestima se mantiene inalterable frente a dichas variables.

f) Existen diferencias significativas entre algunos valores organizacionales y variables tales como lugar de procedencia, género, grado de instrucción y actividades de la empresa; es decir, los valores se alteran en función de dichas variables.

\section{REFERENCIAS BIBLIOGRÁFICAS}

Bonet, J. (1994): Autoestima; Bilbao: Sal Terrae.

Clernes, H. y Bean, R (1996). Cómo desarrollar la autoestima en los niños. Madrid: Debate.

Curwin, RL. (1989). Cómo fomentar los valores individuales. Barcelona: Ceac.

Cruz Ramírez, J. (1996). Autoestima y gestión de calidad. México: Grupo Edit. Iberoamérica.

Lebow, R (1995). La clave de la productividad empresarial. México:

Panorama Editorial.

Loli, A. y López, E. (1997). Autoestima y valores en la calidad y la excelencia. IPSI. Revista de Investigación en Psicología. I(1).

Mora, G. (1995). Valores humanos y actitudes positivas. Bogotá: McgrawHill,. O'Connor, M. (1997). Administración por valores. Bogotá: Grupo Editorial Norma. Robbins, S. P. (1994) Comportamiento organizacional. México: Prentice Hall Hispanoamericana.

Rodríguez, M.(1993). Los valores, clave de la excelencia. México: McgrawHill. Rodríguez, M. y Cols. (SIF). Autoestima: Clave del éxito personal. México: Amecrea. 
